Смагин В. А., Гусеница Я. Н.

V. A. Smagin, Y. N. Gusenitsa

МЕТОД РЕШЕНИЯ ИНТЕГРАЛЬНОГО УРАВНЕНИЯ ВИНЕРА - ХОПФА

ПРИ ГЛАДКИХ РАСПРЕДЕЛЕНИЯХ ВЕРОЯТНОСТЕЙ, СОСТАВЛЯЮЩИХ ЕГО КОМПОНЕНТ

\title{
WIENER-HOPF SOLVER WITH SMOOTH PROBABILITY DISTRIBUTIONS OF ITS COMPONENTS
}

Смагин Владимир Александрович - заслуженный деятель науки РФ, доктор технических наук, профессор, профессор кафедры метрологического обеспечения вооружения, военной и специальной техники Военно-космической академии имени А. Ф. Можайского (г. Санкт-Петербург), E-mail: va smagin@mail.ru.

Mr. Vladimir A. Smagin - Honored Science Worker of the Russian Federation, Doctor of Engineering, Professor, Professor of Faculty of Metrological Maintenance of Arms, Military and Special Equipment, Military Space Academy named after A. F. Mozhaisky (Russia, St.Petersburg). E-mail: va_smagin@mail.ru.

Гусеница Ярослав Николаевич - кандидат технических наук, преподаватель кафедры метрологического обеспечения вооружения, военной и специальной техники Военно-космической академии имени А. Ф. Можайского (г. Санкт-Петербург). E-mail: yaromir226@mail.ru.

Mr. Yaroslav N. Gusenitsa - PhD in Engineering, Lecturer of Faculty of Metrological Maintenance of Arms, Military and Special Equipment, Military Space Academy named after A. F. Mozhaisky (Russia, St.Petersburg) E-mail: yaromir226@mail.ru.

Аннотация. Рассматривается метод приближенного решения интегрального уравнения Винера - Хопфа при гладких распределениях вероятностей, составляющих его компонент. Метод основывается на гипердельтной аппроксимации исходных распределений. Использование в ней преобразования Фурье и характеристической функции позволяет работать в методе со случайными величинами, сосредоточенными на всей вещественной оси абсцисс.

Summary. The Wiener - Hopf solver with smooth probability distributions of its component is presented. The method is based on hyper delta approximations of initial distributions. The use of Fourier series transformation and characteristic function allows working with the random variable method concentrated in transversal axis of absc.

Ключевые слова: интегральное уравнение Винера - Хопфа, гипердельтная аппроксимация распределений, коррекция, преобразование Фурье, характеристическая функция, начальные моменты, скачок распределения функции ожидания.

Key words: Wiener - Hopf integrated equation, hyper delta approximation of distributions, correction, Fourier series transformation, characteristic function, the initial moments, jump of distribution of expectation function.

УДК 519.651

\section{Введение}

К рассматриваемым в настоящей статье задачам по духу и направленности можно отнести достаточно небольшое число публикаций. Для примера отметим некоторые из них.

В [10] рассматриваются вопросы решения интегрального уравнения Винера - Хопфа на основе использования двух вариантов аппроксимации, одна из которых проводится на основе теоремы дискретизации Шеннона. Указываются границы применимости и приложения к задачам статистики.

В [8] рассмотрены новый класс обобщенных вариационных неравенств и новый класс обобщенных уравнений Винера - Хопфа, включающих многозначные и не расширительные отображения в вещественном Гильбертовом пространстве. 
Смагин В. А., Гусеница Я. Н.

МЕТОД РЕШЕНИЯ ИНТЕГРАЛЬНОГО УРАВНЕНИЯ ВИНЕРА - ХОПФА ПРИ ГЛАДКИХ РАСПРЕДЕЛЕНИЯХ ВЕРОЯТНОСТЕЙ, СОСТАВЛЯЮЩИХ ЕГО КОМПОНЕНТ

В статье [9] рассматривается применение методов аппроксимации для решения операторных уравнений, содержащих дискретный оператор Винера - Хопфа. Приводятся оценки погрешности и затухающие свойства решений, получаемых с точки зрения некоторых глад-ких пространств.

В работе [6] рассматриваются некоторые приложения дельта-функции Дирака к решению задач статистики. При этом некоторые известные результаты обобщаются на случай двух переменных.

В докладе [7] рассматривается применение смесей дельта-функций Дирака (гипердельтного распределения) для аппроксимации плотностей функций распределений в аналитической форме. Предлагаются субоптимальные решения и процедура вывода, основанная на последовательном разбиении пространства состояний и размещении компонентов с помощью локальной оптимизации.

В статье [1] приведен пример практического применения описанных выше методов аппроксимации для оценивания надежности программного обеспечения при ограниченном объеме испытаний.

\section{Гипердельтная аппроксимация нормального распределения}

Известно, что нормальное распределение вероятностей может быть представлено дискретным, гипердельтным распределением в аналитическом виде [5]. С учетом трех начальных моментов это представление имеет вид

$$
f(t) \approx C_{1} \Delta\left(t-T_{1}\right)+C_{2} \Delta\left(t-T_{2}\right),
$$

где $f(t)=(1 / \sqrt{2 \pi} \sigma) \exp \left(-(t-m)^{2} / 2 \sigma^{2}\right)-\quad$ плотность вероятности аппроксимируемого распределения; $C_{1}, C_{2}$ - вероятности, $C_{1}>0, C_{2}>0, C_{1}+C_{2}=1 ; \Delta(t)$ - дельта-функция Дирака, а $T_{1}>0, T_{2}>0$ - положительные постоянные величины. Неизвестные значения $C_{i}, T_{i}, i=1,2$ находятся в результате решения четырех нелинейных уравнений, составленных методом моментов. В результате решения получают

$$
f_{a}(t)=\frac{1}{2}(\Delta(t-m+\sigma)+\Delta(t-m-\sigma))
$$

где $m, \sigma$ - параметры аппроксимируемого распределения.

В статье [3] показано, что если хотя бы один из параметров $T_{i}, i=1,2$ принимает отрицательное значение, то есть располагается на отрицательном луче вещественной оси абсцисс, тогда можно составить аналогичную [5] систему нелинейных уравнений. В ней для определения искомых параметров применяются не обычные производные от дельта-функций, а производные от характеристических функций:

$$
\begin{gathered}
C_{1} \varphi_{1}(0)+C_{2} \varphi_{2}=1, \\
C_{1} \frac{\varphi_{1}^{\prime}(0)}{i}+C_{2} \frac{\varphi_{2}^{\prime}(0)}{i}=v_{1}, \\
C_{1} \frac{\varphi_{1}^{\prime \prime}(0)}{i^{2}}+C_{2} \frac{\varphi_{2}^{\prime \prime}(0)}{i^{2}}=v_{2}, \\
C_{1} \frac{\varphi_{1}^{\prime \prime \prime}(0)}{i^{3}}+C_{2} \frac{\varphi_{2}^{\prime \prime \prime}(0)}{i^{3}}=v_{3} .
\end{gathered}
$$

В системе уравнений (1) использованы обозначения характеристической функции и ее производных, а также мнимая единица $i=\sqrt{-1}$.

Система уравнений (1) может иметь как положительные, так и отрицательные значения начальных моментов $v_{j}, j=1-3$. Выражения для дельта-функций в отличие от $\Delta(t-a)$ могут 
принимать вид $\Delta(t+a)$, а функция Хевисайда (для вырожденного распределения вероятностей) вместо $\varphi(t)=e^{i t a}-\varphi(t)=e^{-i t a}$, где $a-$ некоторая вещественная постоянная.

Это позволяет распространить метод аппроксимации вероятностей не только для положительных значений, но и для положительных и отрицательных значений случайной величины. Предложенная коррекция метода основывается на применении вместо преобразования Лапласа преобразования Фурье и характеристической функции.

\section{Пример аппроксимации двух нормальных распределений}

Пусть заданы две плотности вероятности нормального распределения. Первая характеризует время между заявками, поступающими в одноканальную систему массового обслуживания:

$$
a(x)=(1 / \sqrt{2 \pi} \delta) \exp \left(-(x-n)^{2} / 2 \delta^{2}\right)
$$

с параметрами $n=20 h, \delta=5 h$. Вторая характеризует время обслуживания:

$$
b(x)=(1 / \sqrt{2 \pi} \sigma) \exp \left(-(x-m)^{2} / 2 \sigma^{2}\right)
$$

с параметрами $m=10 h, \sigma=3 h$. Требуется представить выражения (2) и (3) в виде гипердельтных распределений. Для распределения (2) будем иметь

$$
a_{a}(x) \approx \frac{1}{2}(\Delta(x-15)+\Delta(x-25)) .
$$

Для распределения (3) получим

$$
b_{a}(x) \approx \frac{1}{2}(\Delta(x-7)+\Delta(x-13)) .
$$

Выражения (4) и (5) получены из формулы, приведенной в [5]:

$$
f(x) \approx \frac{1}{2}(\Delta(x-m+\sigma)+\Delta(x-m-\sigma)) .
$$

Изображения Лапласа (4) и (5) соответственно будут равны:

$$
a_{a}^{*}(s) \approx \frac{1}{2}\left(e^{-15 s}+e^{-25 s}\right), \quad b_{a}^{*}(s)=\frac{1}{2}\left(e^{-7 s}+e^{-13 s}\right),
$$

где $*, s$ - символ преобразования и переменная Лапласа.

\section{Характеристическая функция для формул плотностей вероятностей}

Характеристическая функция плотности вероятности $f(x)$ определяется выражением

$$
\varphi(t)=\int_{-\infty}^{\infty} e^{i t x} f(x) d x,
$$

в котором $i=\sqrt{-1}$. Если случайная величина сосредоточена на положительной полуоси абсцисс, тогда характеристические функции для (2) и (3) принимают вид:

$$
\varphi_{a}(t)=\frac{1}{2}\left(e^{i t(n-\delta)}+e^{i t(n+\delta)}\right), \quad \varphi_{b}=\frac{1}{2}\left(e^{i t(m-\sigma)}+e^{i t(m+\sigma)}\right) .
$$

Если случайная величина сосредоточена на отрицательной полуоси абсцисс, тогда характеристические функции для (2) и (3) имеют вид: 


$$
\begin{aligned}
& \varphi_{a}(t)=\frac{1}{2}\left(e^{-i t(n-\delta)}+e^{-i t(n+\delta)}\right), \\
& \varphi_{b}(t)=\frac{1}{2}\left(e^{-i t(m-\sigma)}+e^{-i t(m+\sigma)}\right) .
\end{aligned}
$$

Характеристическая функция для суммы случайных величин, имеющих плотности вероятностей (2) и (3), определяемая плотностью вероятностей

$$
c_{+}(x)=\int_{0}^{t} b(x-z) a(z) d z
$$

принимает вид

$$
\varphi_{+}(t)=e^{i t(n=m)} \cos (\delta t) \cos (\sigma t)
$$

а характеристическая функция для разности случайных величин, определяемая плотностью вероятностей

$$
c_{-}(x)=\int_{0}^{\infty} b(x+z) a(z) d z \text {, }
$$

принимает следующий вид:

$$
\varphi_{-}(t)=e^{-(n-m) i t} \cos (\delta t) \cos (\sigma t)
$$

\section{Распределение максимума в теории случайных процессов}

Рассмотрим решение задачи о распределении максимума на примере системы массового обслуживания для одноканальной системы $G / G / 1$, приведенное в [2]. Основное рекуррентное соотношение для данной системы массового обслуживания представляется в виде

$$
w_{n+1}=\max \left[0, w_{n}+u_{n}\right]
$$

где $w_{n+1}$ - время ожидания обслуживания поступающего требования в систему с номером $n+1, \mathrm{a}$ $u_{n}=x_{n}-t_{n}-$ разность между временем обслуживания $n$-го требования и промежутком времени между моментами поступления $n$-го и $n+1$-го требований. Обе случайные величины $s_{n}$ и $t_{n}$ независимы от номера $n$ и друг от друга и распределены с плотностями вероятностей $b(t)$ и $a(t)$, а их разность распределена с плотностью вероятности

$$
c(t)=\int_{0}^{\infty} b(t+u) a(u) d u .
$$

Определяемая функция распределения времени ожидания требования в системе при условии, что существует ее стационарный предел, представляется уравнением

$$
W(t)= \begin{cases}\int_{-\infty}^{t} W(t-u) c(u) d u, & t \geq 0 \\ 0, & t<0 .\end{cases}
$$

В книге [2] рассматривались способы преодоления трудностей при изучении системы $G / G / 1$ путем построения приближений и границ для точных решений. Одним из них обсуждалось довольно грубое дискретное приближение. Идея этого подхода состояла в изменении входных распределений $A(t)$ и $B(t)$ таким образом, чтобы основное рекуррентное соотношение 
(8) позволяло получать прямое аналитическое решение для распределения времени ожидания. Итеративное решение этого уравнения достаточно просто, когда обе входные случайные величины представляются дискретными случайными величинами. Они в моменты времени $k \tau$ $(k=0,1,2, \ldots$, с единицей отсчета времени $\tau)$ принимают только ненулевые значения. Тогда можно записать предел рекуррентной процедуры и получить систему линейных разностных уравнений, которая может быть решена методом $z$-преобразований и получено точное распределение времени ожидания.

Но если случайные величины непрерывные, то возникает задача - как непрерывные случайные величины аппроксимировать дискретными таким образом, чтобы сохранялось существо искомого решения. Вопрос о том, как выбрать такое приближение, еще не исследован. Естественная рекомендация состоит в том, чтобы согласовать как больше моментов исходных распределений, начиная с первого момента. Исследование точности такого приближения только начинается [2].

В настоящей статье делается попытка проиллюстрировать этот метод на примере аппроксимации нормальных распределений с точностью до трех начальных моментов. Используя гипердельтное распределение, можно определить моменты скачков входных распределений. Распределения вероятностей в соответствии с (4) и (5) будут равны:

$$
\begin{aligned}
& A(t)=\left\{\begin{array}{cl}
0, & t<n-\delta, \\
\frac{1}{2}, & n-\delta \leq t<n+\delta, \\
1, & t \geq n+\delta,
\end{array}\right. \\
& B(t)= \begin{cases}0, & t<m-\sigma, \\
\frac{1}{2}, & m-\sigma \leq t<m+\sigma, \\
1, & t \geq m+\sigma .\end{cases}
\end{aligned}
$$

Так как берутся только дискретные случайные величины, то можно записать $a(k)=P\left[t_{n}=k \tau\right]$ и $b(k)=P\left[x_{n}=k \tau\right]$. Дискретные функции могут быть представлены и как плотности вероятностей с импульсами в соответствующих точках, как нами было показано в первом разделе статьи.

Если пользоваться уравнением (8), то можно найти распределение вероятностей для $u_{n}$. Определив $c(k)=P\left[u_{n}=k \tau\right]$, так как $u_{n}=x_{n}-t_{n}$, то очевидно, что $c(k)$ в общем виде представляется сверткой [2]:

$$
c(k)=a(-k) * b(i)=\sum_{i=-\infty}^{\infty} a(-k+i) b(i) .
$$

Если $a(k)$ и $b(k)$ содержат небольшое число членов, то свертка легко вычисляется. Но остается нерешенным вопрос о том, как выбрать единицу отсчета времени $\tau$.

Пример 1. Представим плотности исходных распределений (2) и (3), используя формулу (6) в виде дискретных распределений. Эти распределения применительно к свертке (9) показаны на рис. 1. Распределение $a(-k)$ показано на отрицательной полуоси. Для уменьшения размера изображения масштаб по оси абсцисс изменен, численные значения интервалов между дельтафункциями, указанные в (4) и (5), реальным значениям не соответствуют.

Для выполнения рекуррентной процедуры в уравнении (8) предположим, что начальная величина $w_{0}=0$. Кроме того, определим вероятность $p_{n}=P\left[w_{n}=k \tau\right]$. Теперь можно применить рекурсию, состоящую в выполнении операций, описанных уравнением 
Смагин В. А., Гусеница Я. Н.

МЕТОД РЕШЕНИЯ ИНТЕГРАЛЬНОГО УРАВНЕНИЯ ВИНЕРА - ХОПФА ПРИ ГЛАДКИХ РАСПРЕДЕЛЕНИЯХ ВЕРОЯТНОСТЕЙ, СОСТАВЛЯЮЩИХ ЕГО КОМПОНЕНТ

$$
w_{n+1}(y)=\pi\left(w_{n}(y) * c(y)\right.
$$

в котором $\pi$ - оператор, заменяющий плотность вероятности своего аргумента путем замены всей вероятности, связанной с отрицательными значениями, на импульс в точке $y=0$, площадь которого равна этой вероятности. Предельное решение следует из уравнения:

$$
w(y)=\pi(w(y) * c(y) .
$$

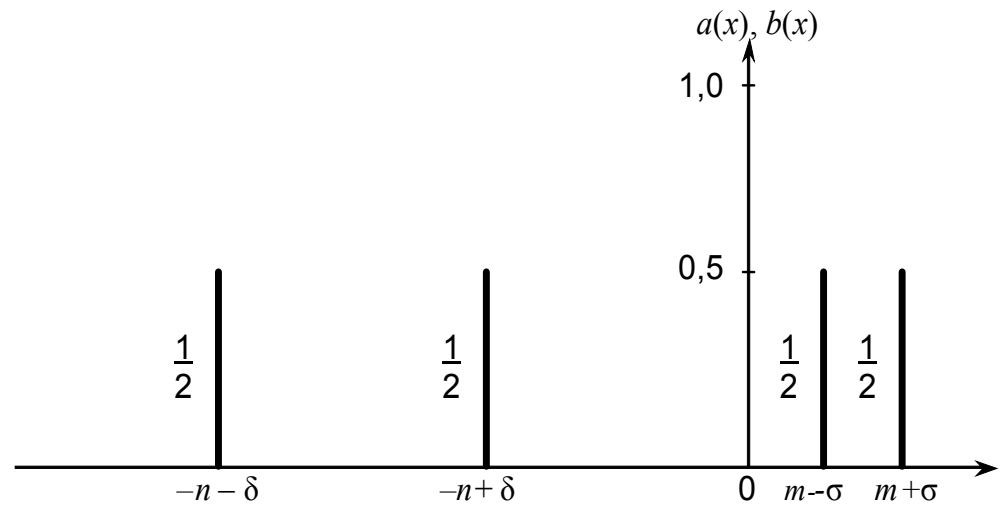

Рис. 1. Дискретное представление исходных распределений

Оно дает стационарную плотность вероятности времени ожидания в системе. Эта плотность должна быть такой, что, когда она образует свертку с $c(y)$ и результирующая плотность переносит свою вероятность с отрицательной оси в импульс, расположенный в нуле, результирующая плотность имеет такой же вид, как и $w(y)$, с которой начиналось рассмотрение.

Для численного расчета примем следующие значения параметров плотностей: для $a(x)$ $n=11 h, \delta=3 h$, а для $b(x) m=10, \sigma=3 h$. В этом случае выражение для свертки (9) принимает вид

$$
c(x)=\frac{1}{4}(\Delta(x-2)+\Delta(x+5)+\Delta(x-7)) .
$$

График этого выражения показан на рис. 2.

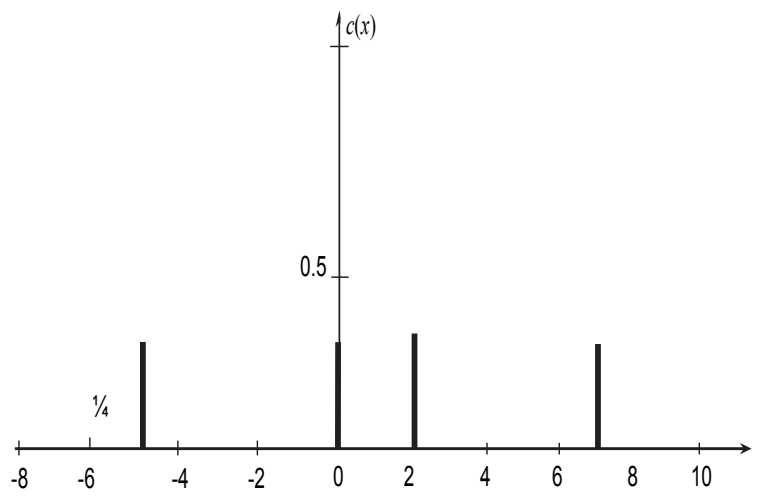

Рис. 2. Графическое изображение дискретной свертки

Далее поступая, как описано в начале примера, и полагая $k=x$, можно записать выражение для определения величины вероятности на $k$-м шаге:

$$
p(k)=\frac{1}{4} p(k+5)+\frac{1}{4} p(k)+\frac{1}{4} p(k-2)+\frac{1}{2} p(k-7), \quad k=1,2,3, \ldots
$$

Граничное уравнение для $p(0)$ равно 


$$
p(0)=\frac{1}{4} p(0)+\frac{1}{4} p(5)+\frac{1}{4} p(-2)+\frac{1}{4} p(-7), \quad k=0 .
$$

Как утверждается в [2], «теперь имеем знакомую задачу решения системы линейных разностных уравнений. К такому же результату приводит метод факторизации Винера. Достоинство описанного здесь метода состоит в том, что он использует в явном виде дискретную природу случайных величин. В обоих случаях трудная часть решения состоит в нахождении корней многочлена [корни знаменателя $P(z)=\sum_{k} p(k) z^{k}$, тогда как в методе факторизации спектра находятся корни выражения $\left.A^{*}(-s) B^{*}(s)-1\right] »$.

В данном разделе статьи показано, как превратить непрерывные задачи в дискретные, для которых применимы довольно простые методы. Это проиллюстрировано решением задачи при обоих нормальных распределениях случайных величин между требованиями и обслуживанием. Однако вопросы о выборе шага дискретизации и адекватности приближения требуют дальнейшего исследования. Здесь мы показали, как переходить от непрерывных распределений к дискретным на основе гипердельтного распределения. Для выполнения численного расчета распределения времени ожидания в системе при любых непрерывных распределениях мы рекомендуем процедуру со сверткой, детально изложенную в [2].

\section{Об определении распределения максимума в непрерывном виде}

Решение уравнения (8) на основе гипердельтной аппроксимации возможно не только в дискретном, но и непрерывном виде. Принцип такого подхода заключается в том, чтобы использовать для решения уравнения (8) не плотности вероятностей исходных распределений $a(x)$ и $b(x)$, а их характеристические функции, а более конкретно - характеристическую функцию их разностной свертки (7). Представляя начальную и все последовательные вероятности состояний $P_{n}$ системы массового обслуживания также характеристическими функциями и осуществляя процесс последовательного их свертывания с характеристической функцией разности исходных случайных величин, а также выполняя на каждом шаге сдвиг отрицательной вероятности в начало координат, получим на положительном луче оси абсцисс характеристическую функцию случайной величины времени ожидания. Для рассмотренных в нем двух нормальных распределений выражение для характеристической функции свертки плотности разности случайных величин будет равно

$$
\varphi(t)=e^{-(n-m) i t} \cos (\sigma t)(\cos \delta t) .
$$

Характеристическая функция начальной единичной вероятности состояния СМО будет принимать вид

$$
P(0)=1
$$

Далее следует применять алгоритм вычисления, аналогичный дискретному алгоритму, рассмотренному в [2].

Шаг 1. Произвести свертку характеристических функций (10) и (11).

Шаг 2. По полученной свертке вычислить вероятность попадания случайной величины на отрицательную часть оси абсцисс. Полученную вероятность сосредоточить в нуле оси абсцисс.

Шаг 3. Получить составную плотность вероятности на положительной полуоси абсцисс и найти для нее характеристическую функцию.

Шаг 4. Умножить полученную характеристическую функцию на характеристическую функцию (10).

Далее шаги 2 - 4 необходимо последовательно повторять до тех пор, пока плотность вероятности на положительной полуоси абсцисс не будет изменяться, то есть совпадать с предшествующей на ней плотностью вероятности. 
Смагин В. А., Гусеница Я. Н.

МЕТОД РЕШЕНИЯ ИНТЕГРАЛЬНОГО УРАВНЕНИЯ ВИНЕРА - ХОПФА ПРИ ГЛАДКИХ РАСПРЕДЕЛЕНИЯХ ВЕРОЯТНОСТЕЙ, СОСТАВЛЯЮЩИХ ЕГО КОМПОНЕНТ

После этого следует использовать полученную плотность вероятности для нахождения стационарной функции распределения времени ожидания обслуживания поступающего требования в систему массового обслуживания.

Вместо исходных нормальных распределений могут использоваться любые распределения, плотности вероятностей которых позволяют получать на основе гиперэкспоненциальной аппроксимации необходимые характеристические функции. Выполнение сверток сводится к перемножению характеристических функций.

Пример 2. Далее мы покажем, как вычислить значения распределения времени ожидания в начале первого и второго циклов - характерные разрывные значения, присущие началу распределения времени ожидания.

Положим, что заданы значения параметров указанных нормальных распределений: $n=11 h$, $\delta=2 h, m=10 h, \sigma=3 h$. Тогда скачок распределения времени ожидания в начале первого цикла определится как $1-\rho_{0}=\int_{-\infty}^{0} c(z) d z$ и численно будет равен $1-\rho_{0}=0,609$. Соответствующая характеристическая функция равна $\varphi(t)=e^{-(n-m) i t} \cos (\sigma t)(\cos \delta t)$. Вычислим начальные моменты $c(x): v_{1}=-1 h, v_{2}=14 h, v_{3}=-40 h$, и решая систему уравнений гипердельтной аппроксимации, найдем значения искомых параметров $C_{1}=0,5, C_{2}=0,5, T_{1}=-4,606 h, T_{2}=2,606 h$. Составим по ней аппроксимационную функцию $F(x)=0,5 \Phi(x-2,606)+0,5 \Phi(x+4,606), \quad$ а затем найдем условную функцию распределения для положительной полуоси $F_{1}(x, \tau)=\frac{F(x+\tau)-F(\tau)}{1-F(\tau)}$.

Обе функции эти показаны на рис. 3.

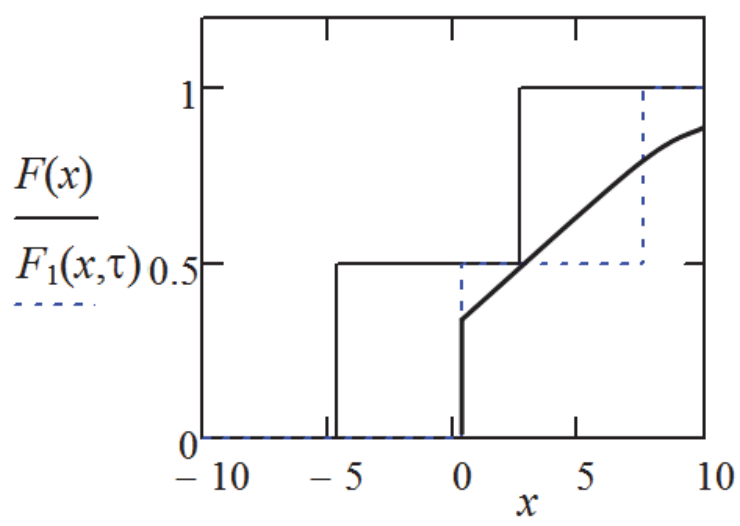

Рис. 3. Аппроксимационная и условная функции распределения

Величина $\tau=-5$. Нетрудно записать выражение для условной плотности вероятности, расположенной на положительной полуоси:

$$
c_{1}(x)=0,5 \Delta(x)+0,5 \Delta(x-7,606) .
$$

Соответствующая (12) характеристическая функция имеет вид:

$$
\varphi_{1}(t)=0,5\left(1+e^{7,606 i t}\right) .
$$

Умножая характеристические функции $\varphi(t)$ и $\varphi_{1}(t)$, получим общую характеристическую функцию

$$
\varphi_{\Sigma}(t)=0,5 \cos (2 t) \cos (3 t) e^{-i t}\left(1+e^{7,606 t}\right) .
$$


Используя (13), найдем значения начальных моментов и параметров новой гипердельтной аппроксимации: $v_{1}=2,802 h ; v_{2}=35,32 h^{2} ; \quad v_{3}=252,96 h^{3} ; \quad C_{1}=0,5 ; \quad C_{2}=0,5 ; \quad T_{1}=-2,438 h$; $T_{2}=8,044 h$.

Запишем выражения для плотности вероятности и функции распределения:

$$
\begin{aligned}
& C(x)=\frac{1}{2}(\Delta(x+2,438)+\Delta(x-8,044)), \\
& C(x)=\frac{1}{2}(\Phi(x+2,438)+\Phi(x-8,044)) .
\end{aligned}
$$

Далее составим условную функцию распределения для положительной полуоси абсцисс и вычислим ее значения при $\theta=-2,438 h$ :

$$
C_{1}(x, \theta)=\frac{C(x+\theta)-C(\theta)}{1-C(\theta)} .
$$

Графики обеих функций представлены на рис. 4.

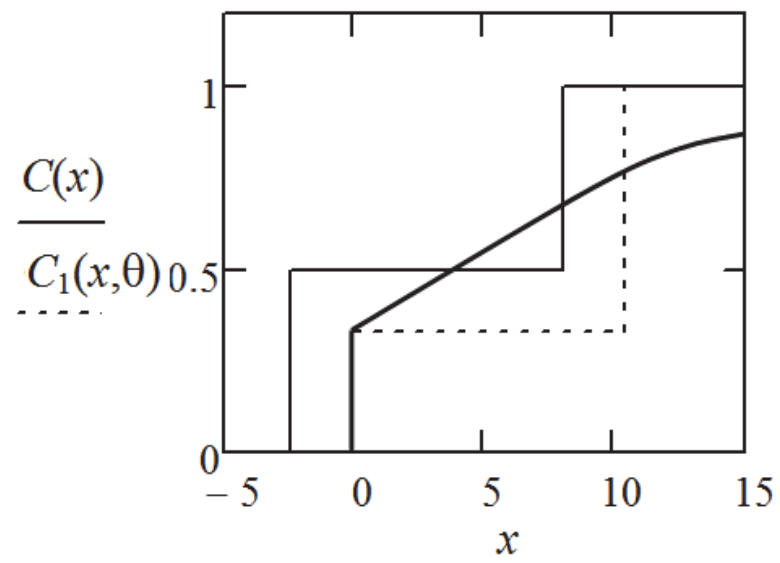

Рис. 4. Функция распределения и условная функция распределения

Начальный скачок функции распределения времени ожидания составит $1-\rho_{1}=C_{1}(0,1 ; 0)=0,333$.

Подобным образом, согласно указанному пошаговому алгоритму, могут быть определены следующие итеративные значения скачков распределения времени ожидания. Более того, при проведении дополнительного исследования, может быть составлен алгоритм вычисления стационарного значения величины скачка функции распределения времени ожидания системы массового обслуживания.

Поведение функции за скачком будет определяться дискретной аппроксимацией функции распределения. В данной статье исследование выполнено только с точностью до трех начальных моментов. Для получения непрерывной функции распределения времени ожидания следует слева направо соединить середины скачков непрерывной плавной линией.

\section{Заключение}

Решению интегрального уравнения Винера-Хопфа распределения максимума случайного блуждания, когда координата процесса может принимать как положительное, так и отрицательное значение, посвящено большое число исследований. Однако представляет определенные трудности его решение, когда оба исходных распределения являются непрерывными и не тривиальными.

Основываясь на идее дискретного решения уравнения, предложенного в книге [2], в настоящей статье развивается дискретный метод решения уравнения именно для непрерывных 
Смагин В. А., Гусеница Я. Н.

МЕТОД РЕШЕНИЯ ИНТЕГРАЛЬНОГО УРАВНЕНИЯ ВИНЕРА - ХОПФА ПРИ ГЛАДКИХ РАСПРЕДЕЛЕНИЯХ ВЕРОЯТНОСТЕЙ, СОСТАВЛЯЮЩИХ ЕГО КОМПОНЕНТ

исходных распределений. В его основе лежит предложенный автором метод гипердельтной аппроксимации произвольных распределений [5]. Но для того, чтобы этот метод был пригоден не только для положительных значений случайной величины, а был распространен на всю вещественную ось, он был скорректирован применением в нем преобразования Фурье и характеристической функции. Это позволило получить итеративное численное решение уравнения Винера-Хопфа при трех начальных моментах обеих нормальных исходных распределениях. Приводятся для иллюстрации решения частные примеры, подтверждающие работоспособность метода.

Метод может быть применен для произвольных гладких распределений вероятностей. Количество начальных моментов, привлекаемых в методе, может быть увеличено. Это приведет к повышению точности решения. Однако численный процесс решения усложнится. Хотя с практической точки зрения иногда ограничение может быть оправдано и малым числом моментов.

Примененный аппарат использования преобразования Фурье и характеристической функции, на наш взгляд, может быть в дальнейшем усовершенствован с целью получения решения уравнения в замкнутом аналитическом виде.

Источник [4] следует рассматривать как дополнительный для ознакомления с предшествующим исследованием авторов в данной области.

\section{ЛИТЕРАТУРА}

1. Гусеница, Я. Н. Обобщенная модель разнотипных программных ошибок для оценивания надежности программного обеспечения / Я.Н. Гусеница // «Наукоемкие технологии в космических исследованиях Земли». - 2015. - Т. 7, № 5. - С. 18-23.

2. Клейнрок, Л. Вычислительные системы с очередями: пер. с англ. / Л. Клейнрок. - М.: Мир. - 1979. - 600 с.

3. Смагин, В. А. Коррекция гипердельтного распределения в теории случайных процессов / В. А. Смагин // Информация и космос. - 2015. - № 4. - С. 60-64.

4. Смагин, В. А. Аппроксимационный метод расчета разомкнутых сетей массового обслуживания В. А. Смагин, Г. В. Филимонихин // АВТ. - 1986. - № 4. - С. 28-33.

5. Смагин, В. А. О моделировании случайных процессов на основе гипердельтного распределения В. А. Смагин, Г. В. Филимонихин // АВТ. - 1990. - № 1. - С. 25-31.

6. Chakraborty, S. Some Applications of Dirac's Delta Function in Statistics for More Than One Random Variable. Applications and Applied Mathematics / S. Chakraborty // An International Journal (AAM). - 2008. - Vol. 3. Pp. $42-54$.

7. Klumpp, V. Dirac Mixture Trees for Fast Suboptimal Multi-Dimensional Density Approximation / V. Klumpp, U. D. Hanebeck // Multisensor Fusion and Integration for Intelligent Systems, Seoul, Republic of Korea. - 2008. Pp. 593-600.

8. Mansotra, P. Wiener - Hopf Equation Technique for Generalized Variational Inequalities and Nonexpansive Mappings / P. Mansotra, B. S. Komal // Applied Mathematical Sciences. - 2012. - No. 18. - Pp. 869-878.

9. Nowak, M. A. Approximation methods for a class of discrete Wiener - Hopf equations / M. A. Nowak // Opuscula Mathematica. - 2009. - Vol. 29, No. 3. - Pp. 271-288.

10. Payandeh Najafabadi, A. T. An Approximate To Solution Of A Subclass Of Wiener - Hopf Integral Equation / A. T. Payandeh Najafabadi, D. Kucerovsky // Proceedings of the World Congress on Engineering - 2009. Vol 2. - Pp. 1262-1267. 